\title{
Early oral feeding after colorectal surgery: a mixed methods study of knowledge
}

\section{translation}

Tayla R. ROBERTSON , ${ }^{1}$ Naomi E. ELDRIDGE, ${ }^{1}$ Megan E. RATTRAY, ${ }^{2}$ Shelley J. ROBERTS,${ }^{2,3,4}$ Ben

DESBROW, ${ }^{2}$ Andrea P. MARSHALL, ${ }^{3,4}$ Azmat B. ALI ${ }^{1}$ and Ingrid J. HICKMAN ${ }^{1,5}$

${ }^{1}$ Department of Nutrition and Dietetics, Princess Alexandra Hospital and 5Mater Research Institute, University of Queensland, Brisbane and ${ }^{2}$ School of Allied Health Sciences and ${ }^{3}$ Menzies Health Institute Queensland, Griffith University and ${ }^{4}$ Gold Coast Health, Gold Coast, Queensland, Australia

\section{Abstract}

Aim: Evidence-based guidelines recommend early oral feeding (EOF) as prescription of an unrestricted diet within 24 hours after colorectal surgery. This study aimed to understand local postoperative feeding practices after colorectal surgery; identify barriers to EOF implementation; select, tailor, and implement stakeholder engagement strategies to facilitate EOF uptake; and evaluate changes to practice.

Methods: A longitudinal, mixed methods study was undertaken, guided by the Knowledge to Action framework. Phase 1 assessed the nature of the problem using postoperative diet audits one and two. In Phase 2, staff interviews identified barriers to EOF implementation. Results from Phases 1 and 2 were fed back to inform Phase 3 strategies. Knowledge uptake was monitored in audits three and four. Phase 4 evaluated outcomes from audit five.

Results: In Phase 1, median time to commencement of full diet was postoperative day four and three in audits one and two respectively. Phase 2 identified EOF barriers, including disparities in diet upgrade practices and variable understanding of hospital diets. In Phase 3, planned strategies were implemented to improve EOF; i) educational session describing local hospital diets; ii) Consultant decision to prescribe a full diet on operation notes; and iii) educational sessions with nursing staff describing changes to EOF practice. In Phase 4, median time to commencement of full diet improved to postoperative day zero. Patients prescribed a full diet on operation notes increased from $0 \%$ to $82 \%$.

Discussion: This study successfully identified and overcame local barriers to improve EOF practices to align with guideline recommendations.

Key words: early oral feeding, enhanced recovery after surgery, postoperative care 


\section{Introduction}

There is well-established evidence to suggest that early oral feeding (EOF) within 24 hours of colorectal surgery is safe and well tolerated ${ }^{1-2}$. EOF may also improve patient outcomes after colorectal surgery with a significant reduction in postoperative complications ${ }^{3-4}$, postoperative infection ${ }^{5}$, length of hospital stay ${ }^{4-}$ ${ }^{5}$, and mortality ${ }^{6}$. As such, EOF recommendations have been incorporated into evidence-based guidelines that encourage the prescription of an unrestricted diet within 24 hours after colorectal surgery ${ }^{1-2,7}$.

Despite established guidelines, the translation of EOF evidence into clinical practice remains an ongoing challenge. Previous literature has described poor adherence to postoperative feeding recommendations, with a higher prevalence of delayed feeding following gastrointestinal procedures ${ }^{8-10}$. Several reasons to explain this discrepancy have been proposed, including a lack of understanding of the potential benefits, a lack of emphasis on nutrition education within surgical training, and the long-standing unfounded dogma of awaiting return of bowel activity before feeding ${ }^{11-12}$.

Effective implementation of enhanced recovery programs requires cultural change to ensure improved communication, teamwork, and organisational support. Programs should be adapted to the local context and deliver a data-driven approach highlighting local deficits, perceived barriers to knowledge uptake, and an opportunity to demonstrate effectiveness ${ }^{13-14}$. Ultimately, engagement of a multidisciplinary team including both front-line and senior staff is inherent to support structural changes for implementation success and sustainability ${ }^{13-16}$.

The aims of this study were to:

1) Understand local postoperative feeding practices after colorectal surgery;

2) Identify barriers and enablers to implementation of EOF guideline recommendations;

3) Select, tailor, and implement stakeholder engagement strategies to facilitate EOF uptake; and

4) Evaluate changes to EOF practices.

\section{Methods}


A longitudinal, mixed methods study, guided by the action cycle of the Knowledge to Action (KTA) framework ${ }^{17}$, was undertaken to evaluate and improve postoperative feeding practices among patients undergoing elective colorectal surgery. The action cycle of the KTA framework represents a plannedaction approach designed to deliberately and effectively translate knowledge into practice ${ }^{17}$. Specifically, it entails; i) identifying the problem and knowledge-to-action gaps; ii) adapting knowledge to the local context; iii) assessing barriers to knowledge use; iv) selecting, tailoring and implementing interventions for knowledge uptake; v) monitoring knowledge use; vi) evaluating outcomes; and vii) sustaining use of knowledge over time. Each phase of the action cycle is influenced by the phases that precede it, outlining a structured yet pragmatic approach used in this study to design, deliver and evaluate EOF practices (see Figure 1).

The study was undertaken on a colorectal ward in a large tertiary metropolitan teaching hospital located in Brisbane, Australia. Enhanced Recovery after Surgery (ERAS) guidelines were not formally in use, rather surgeons prescribed feeding and other postoperative decisions following clinical assessment and consultation. The initial postoperative diet was prescribed by the surgeon in the operation notes at completion of surgery, and changes thereafter occurred during daily morning ward rounds. Integrated electronic medical records (ieMR) and an electronic foodservice management system (CBORD) were in operation at the hospital.

The established ward dietitian was assigned as an EOF champion in June 2016 and was responsible for undertaking the study over the project timeline. De-identified audit data, which included access to hospital medical records, were collected as part of standard review practices within the hospital health service. The study was approved by the local Human Research Ethics Committee (FHREC/17/QGC/101).

Phase 1 - Identifying the nature of the problem Postoperative diet information was retrospectively audited on two occasions in June-July 2016 and February-March 2017 (Figure 1). During this period evidence-based practice guidelines were introduced to the colorectal ward, however no organised intervention was implemented. Audits one and two were completed to assess any changes to practice within a real world clinical setting prior to commencement 
of staff engagement. All consecutive adult patients who underwent an elective bowel resection or stoma reversal were included. Patients were not included in the study if they underwent an emergent procedure or surgery for management of Pseudomyxoma Peritonei (PMP). Patients were excluded from data analysis due to (i) post-operative ICU admission, (ii) return to theatre, or (iii) complicated by prolonged postoperative ileus requiring nasogastric tube decompression.

Postoperative diets for each patient were monitored until a full diet was commenced. This included; (i) the initial diet prescribed by the surgeon in the operation notes at completion of surgery, (ii) the diet received on the ward on postoperative day zero, (iii) the postoperative day the patient was commenced on a free fluid diet, and (iv) the postoperative day the patient was commenced on a full (i.e. unrestrictive) diet. Data were retrieved from online medical records and the hospital electronic foodservice management system. To most accurately reflect dietary changes made during daily surgical ward rounds, the recorded diet code was that which was entered prior to midday each day. Quantitative data were input into Microsoft Office Excel 2003 and analysed using descriptive statistics. All continuous data were tested for normality using GraphPad Prism software $(V 5.03,2009)$ and presented as median [interquartile range (IQR)].

Phase 2 - Adapting knowledge to the local context and assessing barriers to EOF implementation:

Any medical practitioner or nurse directly involved in the care of patients undergoing colorectal surgery at the study site during June 2017 was eligible to participate in semi-structured interviews. Eligible participants were approached by the EOF champion and provided written consent in line with ethics approval. Within this key phase of the KTA cycle, the Theoretical Domains Framework (TDF) was used to develop a semi-structured interview guide (Appendix 1) and gather a more comprehensive understanding of the barriers and enablers surrounding postoperative feeding practices. Consisting of 14 domains, the TDF is a validated, integrative framework that has been widely used to identify influences on health professional behaviour and barriers to practice change within translational research ${ }^{18}$. Interviews were conducted individually on-site at a time convenient to participants, were audio recorded and later transcribed verbatim. There was no pre-conceived hypothesis for why EOF guidelines were not being met 
and so an exploratory approach to inductive thematic analysis ${ }^{19}$ was used to identify emergent themes and then categorise these themes as barriers or enablers to EOF. Themes were crosschecked by secondary analysts and triangulated for consistency and validation of interpretations. Alongside staff interviews, the EOF champion also attended colorectal ward rounds and weekly multi-disciplinary team meetings from June 2017 onwards.

Phase 3 - Selecting, tailoring, implementing stakeholder engagement strategies and monitoring knowledge use:

The content of Phase 3 methodology was informed by the results of Phases 1 and 2. Upon completion of Phases 1 and 2, the EOF champion facilitated a group feedback session with colorectal surgical staff and clinical nurse consultant (CNC) during a multidisciplinary team meeting. Phase 1 audit data identifying the nature of the problem were disseminated alongside barriers and enablers to EOF implementation identified from Phase 2 interview outcomes. The EOF champion facilitated discussion amongst participants, leading to the development of planned education and implementation strategies targeted to improve EOF practices. Knowledge use and practice change during Phase 3 strategies were monitored by audits three and four, completed in June-July 2017 and October-November 2017. Audits were conducted using the same methodology outlined in Phase 1.

Phase 4 - Evaluating outcomes:

Audit five was completed in January-February 2018 to evaluate outcomes upon completion of Phase 3 strategies, and likewise followed the audit methodology outlined in Phase 1.

\section{Results}

Data from 164 patients were included to the five study audits. 34 patients were subsequently excluded from data analysis due to (i) post-operative ICU admission $(n=13)$, (ii) return to theatre $(n=3)$, (iii) complicated by prolonged post-operative ileus requiring nasogastric tube decompression $(n=18)$. Audit data over the study timeline is summarised in Table 1. 
Phase 1 - Identifying the nature of the problem:

During Phase 1, the median time to full diet was postoperative day four (range 2-7 days) and postoperative day three (range 1-7 days) in audits one and two respectively, indicating sustained delay in commencing unrestricted eating after surgery. Patients returning from theatre were typically prescribed a clear fluid or free fluid diet, and upgraded accordingly. In neither audit were patients prescribed a full diet immediately after surgery or on postoperative day zero.

Phase 2 - Adapting knowledge to the local context and assessing barriers to EOF implementation: Sixteen staff participated in interviews, lasting 15-20 minutes each. Staff included six surgical Consultants, one surgical Fellow, two surgical trainees (Principal House Officer/Registrar). three junior medical doctors (two interns and one Junior House Officer), one clinical nurse consultant (CNC), one clinical nurse, and two registered nurses (RNs). All colorectal Consultants on staff completed interviews. Six key themes were identified from the interview results; two manifesting as enablers and four as barriers to EOF.

a) Broad awareness and strong cultural acceptance of the benefit of early oral feeding Among colorectal Consultants, there was widespread awareness of changing historical practices and support for ERAS initiatives and similar fast track surgical pathways recommending EOF after surgery.

- If it's in those protocols then it must be safe... and with good evidence supporting it. So I'm a big fan of early oral feeding." (Consultant 1)

- "So early oral feeding is the way of... when I was training it was the way of the future, and now it's the way of the present because we know that early oral feeding leads to shorter length of stay, early physiological return to normal, and essentially providing the gut with its trophic feeding". (Consultant 2)

b) Wide acceptance of an elective surgery EOF pathway with ability to opt-out for complicated cases Consistent with practice guidelines, colorectal surgical staff were supportive of implementing EOF in uncomplicated elective colorectal patients as this was deemed best practice surgical care. Emphasis was 
placed on the education process surrounding patients and ward staff to ensure the implementation process was accurately communicated and any patients experiencing complications were identified and managed appropriately.

- "I think a pathway is a good idea... but there has to be an education process... it's education of nursing staff and education of junior doctors." (Consultant 3)

- "I think it would be good, but we also need to recognise those who should be pulled back from the pathway... so those showing signs of gastroparesis or ileus." (Consultant 4)

c) Decision makers use a pragmatic approach to diet upgrades

Current diet upgrade decisions were guided by a pragmatic approach based on the patient clinical assessment at the time of review. Nausea and vomiting were dominant symptoms influencing this decision-making process, as well as patient-reported factors such as 'feeling well' and 'feeling hungry'. Greater caution was reported with elderly patients due to increased risk of aspiration pneumonia. Factors at the time of surgery, such as the amount of bowel handling and type and length of anaesthetic, were also considered. Historical clinical indicators influencing diet upgrade decisions, such as 'bowel sounds' and 'bowels opening', had been generally abandoned by Consultants, but consideration of these factors still remained with junior medical staff and nursing staff.

- "If you see them day one post-op and they're nauseous and vomiting you don't want to push them too hard. And especially in the elderly population, you don't want them to aspirate." (Colorectal Fellow)

- "I find one of the most reliable things is when patients actually complain of being hungry and are interested in food. I think that's usually a sign that... you're probably under-feeding them." (Consultant 5)

d) Disparities exist in diet upgrade practices

The speed of diet upgrades was often dependent on the individual surgeon's training and education. While Consultants remained the dominant influence on diet upgrade advice, the surgical Fellow and 
Registrars were more commonly the decision makers at the ward level and would often err on the side of caution. Junior medical staff were ill-equipped and lacked confidence to make decisions about diet upgrades in the absence of senior clinicians.

- II'm sure the guys who are very reluctant to start oral feeding too early is just because that was the way they were taught and they haven't had any issues with it, so why would they change." (Consultant 1)

- "When we do handover I ask "what diet is this guy on?" and the interns aren't always sure...that's not something that's mentally being flagged in their mind... I guess when you're a junior doctor it's hard to be across every detail of the patient" (Principal House Officer)

Consultants often perceived diet upgrades to occur faster than they do in practice. After documenting a postoperative diet in the operation notes, Consultants were not typically involved in further diet upgrade decisions in uncomplicated patients. From their experience at a ward level, junior medical staff reported a traditional diet upgrade process from clear fluids to free fluids to a full diet.

- "My postoperative notes say they can start free fluids when they're awake and alert... and I upgrade to a normal diet the next day." (Consultant 6)

- "People tend to go straight to clear fluids, and then sort of stepwise up... so clear fluids, free fluids, diet as tolerated." (Intern 1)

e) Limited understanding of the nature and availability of different hospital diets There was confusion and a limited understanding of hospital diets, largely driven by non-standardised diet codes across different hospitals where surgical Consultants were employed. While Consultants correctly differentiated clear fluid from free fluid diets, there was no clear consensus on the rationale for using one diet code over another postoperatively. Some Consultants reported a preference for a 'light ward diet', however there was a lack of consensus regarding what a 'light' diet included and how it differed from a standard full diet.

- "I don't know whether there's just a bit of hocus pocus... or whether there's really any difference with giving someone clear fluids or free fluids." (Registrar) 
- "I don't use clear fluids very much because I don't think there's much difference between clear fluids and free fluids. Clinically for the patient it's going to be fairly liquid by the time the stomach deals with it, and so I don't think there's much use for clear fluids." (Fellow)

- "I suppose you should have a 'not that rich' diet... not spicy... that's I suppose what I mean by a light ward diet." (Consultant 5)

g) Diet upgrades are not systematically tracked and are vulnerable to communication breakdown 'Current diet' appeared to be a greater influence than 'total days on diet' when determining diet upgrades during ward rounds. Staff relied on memory, previous ward round notes, or team lists to identify current patient diets, and the number of days on fluid only diets was not documented as a standard component of this process. There was a lack of consistency regarding the nursing role in diet advocacy.

- "They don't really [track diet upgrades]... you just follow the notes. And things do get missed because the last note you've read said this and then they meant to say upgrade but haven't." (Registered Nurse 1)

- "It's all very well for you to see the patient and make a decision, but then that has to be communicated to nursing staff who have to understand it, not be on a break, and then they might forget, or the message gets changed." (Consultant 3)

Phase 3 - Selecting, tailoring, implementing stakeholder engagement strategies and monitoring knowledge use:

As described previously, Phase 3 strategies were informed by results from Phases 1 and 2. Following discussion facilitated by the EOF champion, three planned education and implementation strategies were developed to improve EOF practices.

The first strategy was an educational session with colorectal surgical staff and CNC about the nature and availability of different hospital diet codes, as this was identified from interview outcomes as a limitation and potential barrier to implementing EOF guideline recommendations. The EOF champion presented a 
summary of the common diet codes available at the hospital, including clear fluid, free fluid, full, and high protein high energy diets. A pictorial guide, examples of meal trays, and taste testing accompanied the education.

The second approach was to change the wording of surgical operation notes to prescribe a full diet on postoperative day zero for all uncomplicated elective colorectal patients. This was decided among five of the six Consultants during the group feedback session as it was deemed best practice surgical care and an uncomplicated strategy to implement.

Thirdly, educational sessions were carried out with ward nursing staff, facilitated by the EOF champion, colorectal CNC, and colorectal ward clinical facilitator. Over three consecutive weeks, $>80 \%$ of nursing staff on the colorectal ward attended educational sessions. Similar to feedback provided to surgical staff, sessions included: a summary of practice guideline recommendations of an unrestricted diet within 24 hours postoperatively; and phase 1 audit data identifying a discrepancy between guidelines and current practice. Communicating the surgical team's decision for elective colorectal patients to be prescribed a full diet on postoperative day zero on surgical notes was also included in the nursing education session. Nursing staff were educated to encourage patients to eat meals as tolerated and escalate any concerns of increasing nausea or vomiting to the surgical staff.

Audits three and four monitored knowledge use and practice change. The median time to full diet was postoperative day two (range 0-5 days) and postoperative day one (range 0-3 days) in audits three and four respectively. The number of patients prescribed a full diet on the surgical operation notes was $12 \%$ in audit three and $61 \%$ in audit four (Table 1 ).

Phase 4 - Evaluating outcomes:

In audit five, the median time to full diet was postoperative day zero (range $0-5$ days), with $82 \%$ of patients prescribed a full diet on the surgical operation notes (Table 1). 


\section{Discussion}

This study utilised the action cycle of the KTA framework to; understand local postoperative feeding practices; identify barriers and enablers to implementation of EOF guideline recommendations; select, tailor, and implement stakeholder engagement strategies to facilitate EOF uptake; and evaluate changes to EOF practices. Over the study timeline, there was a consistent and sustained improvement in median time to full diet prescription, improving from postoperative day four to postoperative day zero. This change in practice can be attributed to application of the KTA framework to design and deliver strategies specifically targeted to overcome barriers and translate knowledge into practice within a real world clinical setting.

A key component of this study included staff interviews, which identified pre-existing decision-making processes and local barriers to EOF implementation. While Consultants were typically responsible for prescribing postoperative diets in the operation notes at the time of surgery, the surgical Fellow or Registrar guided subsequent diet upgrades thereafter. Diet upgrade decisions typically followed a pragmatic approach based on clinical assessment, however, were usually influenced by the individual surgeon's training and education. Staff interviews also identified a limited understanding of the diet codes available at the hospital, and lack of consensus among surgical staff on the rationale for prescribing particular diets. Collectively, these barriers highlighted a need to address EOF education and available diet codes as a necessary component of the implementation strategy. This was consistent with previous literature that has emphasised the importance of staff-related education to facilitate initiation of change and transition of practice prior to implementation of enhanced recovery programs ${ }^{11-16,20}$. As such, this study included education sessions that described EOF guideline recommendations and descriptions of the nature and availability of local hospital diet codes.

Another recurring theme evident in previous literature was the need for communication and collaboration throughout the surgical staff hierarchy and multidisciplinary team ${ }^{13,15}$. Interestingly, initial improvements in median time to commencement of full diet were noted when the EOF champion began attending colorectal ward rounds and weekly multi-disciplinary team meetings, as well as commencing staff 
interviews. This process of engagement was another important catalyst to instigate practice change, which relayed an initial positive effect onto EOF improvements as illustrated in audit three. The role of staff engagement and effective team communication has also been previously described as a necessary enabler for successful implementation of enhanced recovery programs ${ }^{11-16,20}$.

Stakeholder engagement strategies within Phase 3 of the study were designed and implemented at the Consultant level to drive effective and sustainable change. Consultant decision to prescribe a full diet on postoperative day zero for all uncomplicated elective patients reflected a pragmatic approach to practice previously identified from interview results. Whilst the decision was not unanimous across the whole surgical team (5 of 6 Consultants agreed), it indicates that positive change can occur whilst maintaining flexibility in case-by-case decision making for individual surgeons. Revisiting this issue with the whole surgical team when longer-term outcome and complication data is available will be of interest. Overall efficacy of EOF uptake among Consultants was evident from audit results where the number of patients prescribed a full diet on operation notes increased progressively from $0 \%$ to $82 \%$. The importance of type of dietary provision has been described previously, where postoperative patients receiving a fluid only diet were significantly more likely to consume $<50 \%$ of their estimated energy requirements compared to those receiving a non-fluid (full or similar) $\operatorname{diet}^{21}$. Implementing system strategies such as this was designed to elicit organisational change and overcome sustainability limitations commonly reported when champions withdraw from a project ${ }^{22}$.

Two key limitations were identified within the current study. Firstly, no strategy was established to ensure diet upgrades were systematically tracked. While this was a barrier identified in staff interviews, the decision to prescribe patients a full diet on postoperative day zero negated the need for tracking postoperative diet upgrades. Despite this, systematic tracking of diets is recommended to ensure patients with emergency surgeries or postoperative complications are upgraded to a nutritionally adequate diet in a timely manner. Secondly, the high level of evidence supporting EOF after colorectal surgery may have supported cultural change to a greater extent than what may be experienced for other acute conditions and therefore limit the generalisability of this study design in the absence of similar high quality literature 
to support the implementation. Despite this, the methodology reported in this study remains applicable for use in other facilities with colorectal surgical units. Whilst the exact content of Phase 3 strategies may be site-specific, the methodology process developed from the action cycle of the KTA framework can be replicated to improved EOF practices within other facilities.

Finally, there is well-established literature to suggest that an ongoing process of audit and feedback can increase compliance with guideline recommendations and improve project sustainability ${ }^{17,23}$. A future direction of this study is to develop a plan for annual audit and feedback, including multidisciplinary accountability for outcome measures and audit dissemination. Consistent with the KTA cycle, knowledge sustainability may present new barriers different to those encountered at the time of initial knowledge use. Project sustainability should hence continue to cycle through the KTA feedback loop as new barriers emerge.

In conclusion, this study has successfully identified and overcome local barriers to improve EOF practices to align with guideline recommendations. Consistent with the KTA implementation framework, future long-term audit and feedback strategies are recommended to sustain knowledge use.

\section{Acknowledgements}

The authors would like to thank the contribution of staff and patients of the colorectal ward at the Princess Alexandra Hospital in Brisbane Australia.

\section{References}

1. Weimann A, Braga M, Carli F et al. ESPEN guideline: clinical nutrition in surgery. Clin Nutr 2017; 36: $623-50$.

2. Gustafsson UO, Scott MJ, Schwenk W et al. Guidelines for perioperative care in elective colonic surgery enhanced recovery after surgery (ERAS) society recommendations. World J Surg 2013; 37: $259-84$. 
3. Osland E, Yunus RM, Khan S, Memon MA. Early versus traditional postoperative feeding in patients undergoing resectional gastrointestinal surgery: a meta-analysis. JPEN 2011; 35: 473-87.

4. Zhuang C-L, Ye X-Z, Zhang C-J, Dong Q-T, Chen B-C, Yu Z. Early versus traditional postoperative oral feeding in patients undergoing elective colorectal surgery: a meta-analysis of randomized clinical trials. Dig Surg 2013; 30: 225-32.

5. Lewis SJ, Egger M, Sylvester PA, Thomas S. Early enteral feeding versus "nil by mouth" after gastrointestinal surgery: systematic review and meta-analysis of controlled trials. J Gastrointest Surg 2001; 323: 773-76.

6. Lewis SJ, Andersen HK, Thomas S. Early enteral nutrition within $24 \mathrm{~h}$ of intestinal surgery versus later commencement of feeding: a systematic review and meta-analysis. J Gastrointest Surg 2008; 13: 569-75.

7. Carmichael JC, Keller DS, Baldini G. Clinical practice guidelines for enhanced recovery after colon and rectal surgery from the American Society of Colon and Rectal Surgeons Society of American Gastrointestinal and Endoscopic Surgeons. Dis Colon Rectum 2017; 60: 761-84.

8. Rattray M, Roberts S, Marshall A, Desbrow B. A systematic review of feeding practices among postoperative patients: is practice in-line with evidence-based guidelines? J Hum Nutr Diet 2018; 31: $151-67$

9. Rattray M, Marshall A, Desbrow B, Roberts S. Feeding practices and nutritional intakes among non-critically ill, postoperative adult patients: An observational study. NCP 2018 doi: 10.1002/ncp.10103.

10. Byrnes A, Banks M, Mudge A, Young A, Bauer J. Enhanced Recovery After Surgery as an auditing framework for identifying improvements to perioperative nutrition care of older surgical patients. Eur J Clin Nutr 2018; 72: 913-16.

11. Martindale RG, McClave SA, Taylor B, Lawson CM. Perioperative nutrition: what is the current landscape? JPEN 2013; 37: 5S-20S.

12. Frantz DJ, Munroe C, McClave SA, Martindale R. Current perception of nutrition education in US medical schools. Curr Gastroenterol Rep 2011; 13: 376-79. 
13. Stone $A B$, Yuan $C T$, Rosen $M A$ et al. Barriers to and facilitators of implementing enhanced recovery pathways using an implementation framework, a systematic review. JAMA Surg 2018; 153: $270-79$.

14. Steenhagen E. Enhanced recovery after surgery: it's time to change practice! Nutr Clin Pract 2015; 31: 18-29.

15. Lyon A, Soloman MJ, Harrison JD. A qualitative study assessing the barriers to implementation of enhanced recovery after surgery. World J Surg 2014; 38: 1374-80.

16. Maessen J, Dejong CHC, Hausel J et al. A protocol is not enough to implement an enhanced recovery programme for colorectal resection. Br J Surg 2007; 94: 224-31.

17. Graham I, Logan J, Harrison M et al. Lost in knowledge translation: time for a map? J Contin Educ Health Prof 2006; 26: 13-24.

18. Cane J, O'Connor D, Michie S. Validation of the theoretical domains framework for use in behaviour change and implementation research. Implement Sci 2012; 7: 37.

19. Guest G, MacQueen KM, Namey EE. Applied Thematic Analysis. California: SAGE Publications, 2012.

20. Kahokehr A, Sammour T, Zargar-Shoshtari K, Thompson L, Hill AG. Implementation of ERAS and how to overcome the barriers. Int J Surg 2009; 7: 16-19.

21. Byrnes A, Worrall J, Young A, Mudge A, Banks M, Bauer J. Early post-operative diet upgrade in older patients may improve energy and protein intake but patients still eat poorly: an observational pilot study. J Hum Nutr Diet doi: 10.1111/jhn.12572.

22. Conn LG, McKenzie M, Pearsall EA, McLeod RS. Successful implementation of an enhanced recovery after surgery programme for elective colorectal surgery: a process evaluation of champions' experiences. Implementation Science 2015; 10: 99.

23. Jamtvedt G, Young JM, Kristoffersen DT et al. Audit and feedback: effects on professional practice and health care outcomes. Cochrane Database Syst Rev 2006; 2: CD000259. 
Appendix 1

Staff interview guide

"Before we start the interview, can I check that you have around 10-15 minutes to talk to me today and that you are happy for the interview to be audio recorded? The focus of this interview is on your perspectives of and experience with early oral feeding after surgery. Your answers will remain confidential and will be anonymous if used in reporting."

Early Oral Feeding is defined as the provision of nutritionally significant oral or enteral nutrition given within 24 hours postoperatively.

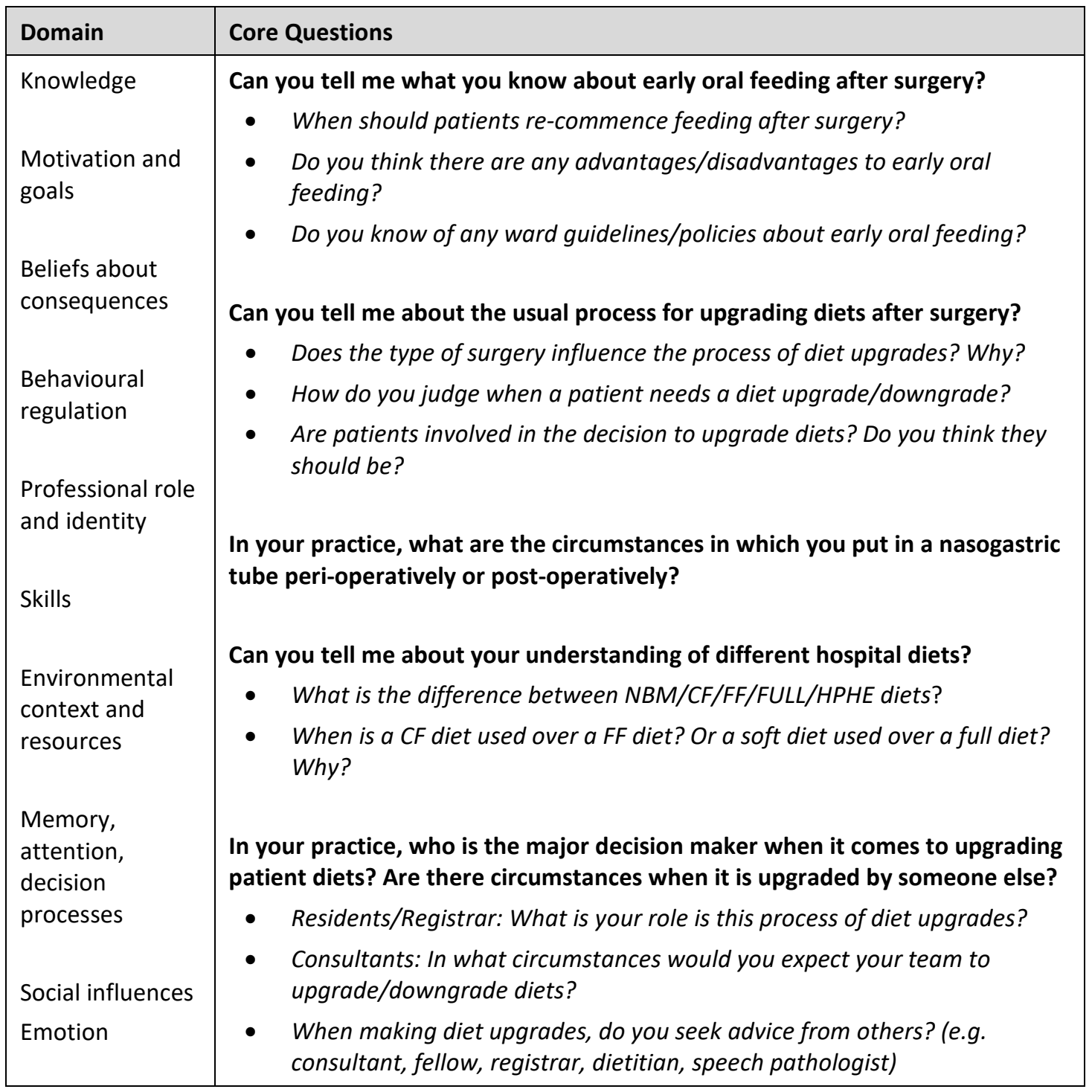




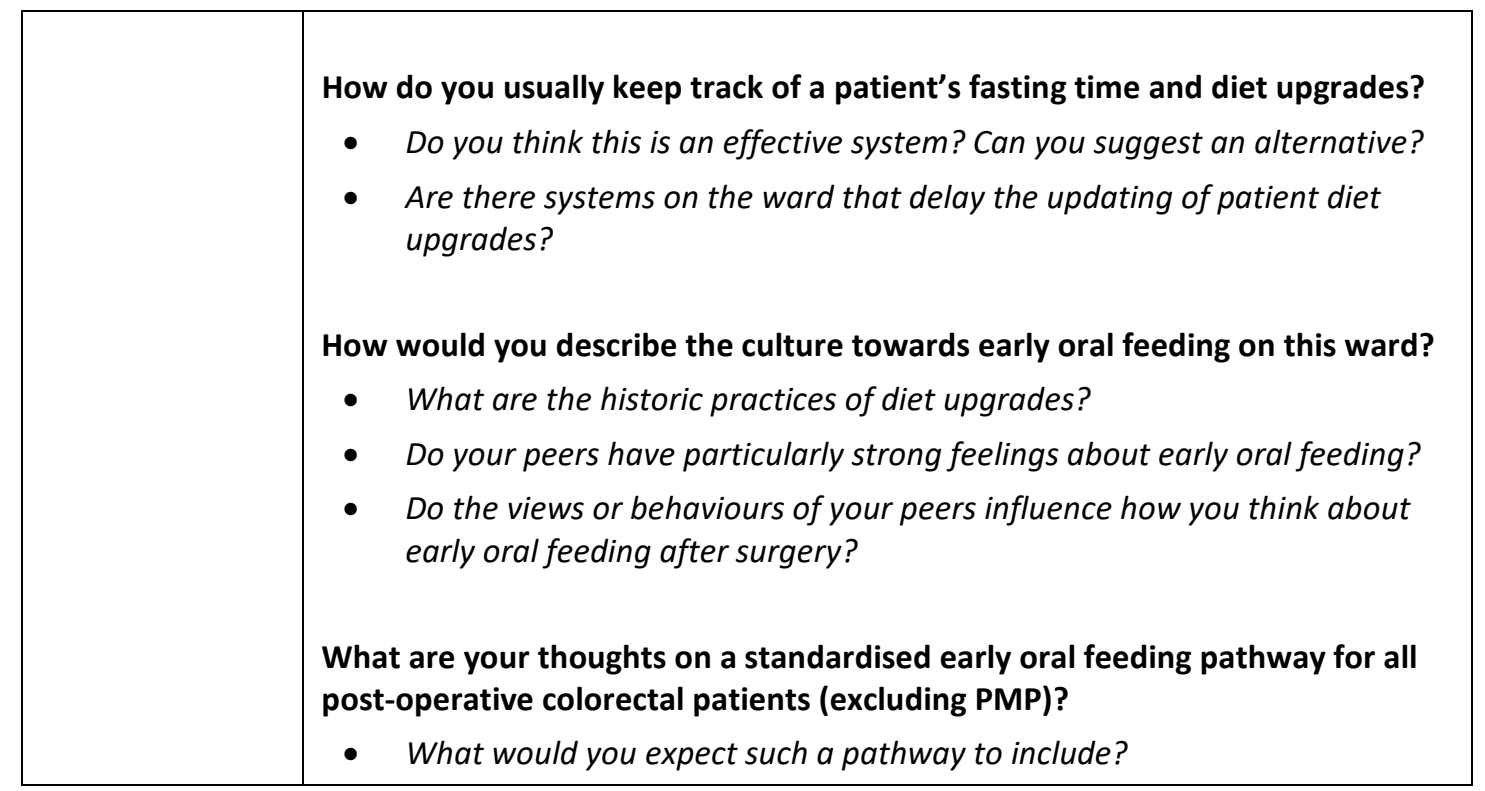

Conclusion: "We've come to the end of my questions, thank you very much for your time. Please do not hesitate to contact me if you have any further questions." 
Table 1: Key measures of EOF practice across five audits over an 18-month period

\begin{tabular}{|c|c|c|c|c|c|}
\hline & \multicolumn{2}{|c|}{ Phase 1} & \multicolumn{2}{|c|}{ Phase 3} & \multirow[b]{2}{*}{$\begin{array}{c}\text { Phase } 4 \\
\text { Audit } 5 \\
\text { Feb-Mar } \\
2018 \\
(n=28)\end{array}$} \\
\hline & $\begin{array}{c}\text { Audit } 1 \\
\text { Jun-Jul } \\
2016 \\
(n=23)\end{array}$ & $\begin{array}{c}\text { Audit } 2 \\
\text { Feb-Mar } \\
2017 \\
(n=20)\end{array}$ & $\begin{array}{c}\text { Audit } 3 \\
\text { Jun-Jul } \\
2017 \\
(n=31)\end{array}$ & $\begin{array}{c}\text { Audit } 4 \\
\text { Oct-Nov } \\
2017 \\
(n=28)\end{array}$ & \\
\hline $\begin{array}{l}\text { Postoperative day } \\
\text { commenced on a full diet } \\
\text { - median (range) }\end{array}$ & $4(2-7)$ & $3(1-7)$ & $2(0-5)$ & $1(0-3)$ & $0(0-5)$ \\
\hline $\begin{array}{l}\text { Number of patients } \\
\text { prescribed a free fluid or } \\
\text { clear fluid diet on the } \\
\text { operation note }-n(\%)\end{array}$ & 23 (100\%) & 20 (100\%) & 27 (87\%) & 11 (39\%) & 5 (18\%) \\
\hline $\begin{array}{l}\text { Number of patients } \\
\text { prescribed a full diet on the } \\
\text { operation note }-n(\%)\end{array}$ & $0(0 \%)$ & $0(0 \%)$ & $4(12 \%)$ & 17 (61\%) & $23(82 \%)$ \\
\hline $\begin{array}{l}\text { Number of patients who } \\
\text { received a full diet on } \\
\text { postoperative day } 0-n(\%)\end{array}$ & $0(0 \%)$ & $0(0 \%)$ & $3(10 \%)$ & $12(43 \%)$ & 20 (71\%) \\
\hline
\end{tabular}




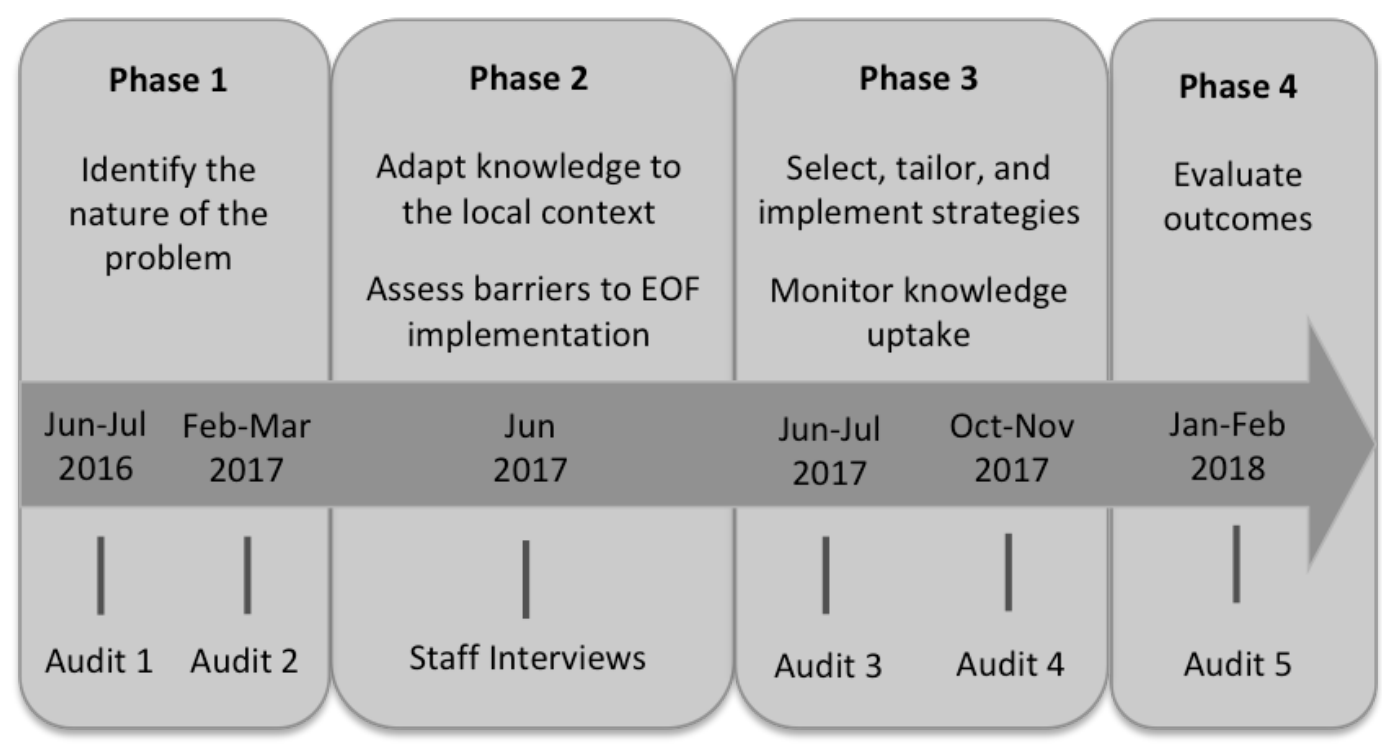

Figure 1: Schematic diagram of study design. Phases align with the action cycle of the KTA framework: Phase 1 identified the nature of the problem; Phase 2 adapted knowledge to the local context and assessed barriers to EOF implementation; Phase 3 selected, tailored, and implemented stakeholder engagement strategies to improve EOF and monitored knowledge uptake; and Phase 4 evaluated outcomes. 